\title{
Upper Bound Estimation of Fractal Dimension of Fractional Calculus of Continuous Functions
}

\author{
Li Yang and Liang Yongshun* \\ Institute of Science, Nanjing University of Science and Technology, Nanjing, 210094, China \\ Email: liangyongshun@tom.com
}

\begin{abstract}
In the present paper, upper bound estimation of upper Box dimension of RiemannLiouville fractional integral of order $v$ of any continuous functions on a closed interval has been proved to be no more than $2-v$ when $0<v<1$. If a continuous function which satisfies $\alpha$-Hölder condition on a closed interval, upper Box dimension of its Riemann-Liouville fractional integral is no more than $2-\alpha$ when $0<\alpha<1$. Upper bound of upper Box dimension of Riemann-Liouivlle fractional integral of certain type of fractal functions has been proved to be no more than Box dimension of functions themselves.
\end{abstract}

Keywords: Box dimension, Riemann-Liouville fractional integral, fractal function, upper bound, length of graph.

\section{Introduction}

Estimation of fractal dimension of fractional calculus of continuous functions, such as Weierstrass function[1], Bescovitch function[10] and other fractal functions has been a goal of much recent research. First work maybe Refs.[9,13] which discussed pictures and numerical results of fractional calculus of certain fractal curves such as Von Koch curve. Ref.[11] investigated fractal dimension of Riemann-Liouville fractional integral and derivative of Weierstrass function. The linear relationship between fractal dimension and orders of fractional calculus has been set up. Fractal dimension of Riemann-Liouville fractional calculus of Besicovitch function had been investigated in Ref.[2]. Other conclusions of fractal dimension of fractional calculus of continuous functions can be found in Refs. [3,8,12,14]. Fractal dimension of fractional integral of most functions seems no more than fractal dimension of functions themselves.

Though problems of fractal dimension of fractional calculus of fractal functions with certain expressions have been extensively studied by authors, it seems to us that little has been known on fractal dimension of fractional calculus of a type of fractal functions which have no expressions. In other words, those functions discussed above often have good properties and special expressions, while there is little work about fractional calculus of functions of certain fractal dimension without expression. Ref.[4] made research on Riemann-Liouville fractional integral of continuous functions with bounded variation. Fractal dimension of Riemann-Liouville fractional integral of any order of such functions has been proved to be 1. Fractal dimension of Riemann-Liouville fractional integral of certain 1-dimensional continuous fractal functions with unbounded variation on closed intervals have been discussed in Ref.[5].

Upper to now, most work discuss fractal dimension of fractional calculus of special functions such as Weierstrass function, Besicovitch function, self-affine function, fractional interpolation functions, Radmacher series, special fractal curves and certain complex-expressed fractal functions. The present paper consider fractal dimension of fractional integral of a type of continuous functions which have no expressions on closed intervals. Object of the paper is to prove that upper bound of upper Box dimension of Riemann-Liouville fractional integral of a type of continuous functions on closed intervals is no more than Box dimension of functions themselves.

In the present paper we use Box dimension, upper Box dimension and lower Box dimension which are defined as the following Definition 1.1 to describe continuous functions or fractal functions on any closed intervals.

Definition 1.1.[1,10] Let nonempty set $F$ be a bounded subset of $\mathbb{R}^{2}$ and $N_{\delta}(F)$ be the smallest number of sets of diameter at most $\delta$ which can cover $F$. Lower Box dimension and upper Box dimensions of $F$ 
respectively are defined as

$$
\underline{\operatorname{dim}}_{B}(F)=\lim _{\delta \rightarrow 0} \frac{\log N_{\delta}(F)}{-\log \delta}
$$

and

$$
\overline{\operatorname{dim}}_{B}(F)=\varlimsup_{\delta \rightarrow 0} \frac{\log N_{\delta}(F)}{-\log \delta} .
$$

If (1.1) and (1.2) are equal we refer to the common value as Box dimension of $F$

$$
\operatorname{dim}_{B}(F)=\lim _{\delta \rightarrow 0} \frac{\log N_{\delta}(F)}{-\log \delta} .
$$

Form Definition 1.1, we know upper Box dimension of any continuous functions on a closed interval is no more than 2. Lower Box dimension of any continuous functions on a closed interval is no less than 1. In other words, if Box dimension of a continuous function on a closed interval exists, it is no more than 2 and no less than 1.

With the following definition of Riemann-Liouville fractional integral, we can discuss fractal dimension of Riemann-Liouville fractional integral of any continuous functions on closed intervals.

Definition 1.2[6,7]. Let $f(x)$ be a continuous function. $v>0$ and $D^{-v} f(0)=0$, we call

$$
D^{-v} f(x)=\frac{1}{\Gamma(v)} \int_{0}^{x}(x-t)^{v-1} f(t) \mathrm{d} t
$$

Riemann-Liouville integral of $f(x)$ of order $v$.

Now we are going to investigate upper Box dimension of Riemann-Liouville fractional integral of any continuous functions on closed intervals. Especially, when a continuous function $f(x)$ on a closed interval satisfies Hölder condition which is defined as Definition 1.3, we want to know upper Box dimension of Riemann-Liouville fractional integral of $f(x)$ decreases or not.

Definition 1.3.[1] Let $f(x)$ be a continuous function on $[0,1]$ and $\alpha \in(0,1)$. If

$$
|f(x)-f(y)| \leq C|x-y|^{\alpha}, \quad x, y \in[0,1]
$$

where $C$ is a positive constant, we say $f(x)$ satisfies $\alpha$-Hölder condition.

Throughout the present paper, all functions are continuous on the unit interval $I=[0,1] . \Gamma(f, I)$ denotes the graph of a function $f(x)$ on $I$. $C$ is a positive constant that may have different values at different occurrences even in the same line. $C_{[0,1]}$ denotes all continuous functions on $I$ and $C_{[0,1]}^{\alpha}$ is the set of all continuous functions satisfying $\alpha$-Hölder condition on $I$.

\section{Upper Bound of Upper Box Dimension of Riemann-Liouville Fractional Integral of $f(x) \in C_{[0,1]}$}

In this section, we make research on upper Box dimension of Riemann-Liouville fractional integral of any continuous functions on $I$. Estimation of upper bound of upper Box dimension of Riemann-Liouville fractional integral functions has been got. If a continuous function satisfies $\alpha$-Hölder condition which is given in Definition 1.3, upper Box dimension of its Riemann-Liouville fractional integral is no more than $2-\alpha$. By Ref.[1], it can also be written as the following Lemma 2.1.

Lemma 2.1[1]. Let $f(x) \in C_{[0,1]}^{\alpha}$. Then,

$$
\overline{\operatorname{dim}}_{B} \Gamma(f, I) \leq 2-\alpha .
$$

If $f(x) \in C_{[0,1]}$, for $0<v<1$, Riemann-Liouville fractional integral of $f(x)$ of order $v$ is given as follows.

$$
D^{-v} f(x)=\frac{1}{\Gamma(v)} \int_{0}^{x}(x-t)^{v-1} f(t) \mathrm{d} t
$$


We know $\Gamma\left(D^{-v} f, I\right)$ denotes the graph of $D^{-v} f(x)$ on $I$. With Lemma 2.1, we have the following conclusion which means upper Box dimension of Riemann-Liouville fractional integral of any continuous functions on $I$ is no more than $2-v$.

Theorem 2.2. Let $f(x) \in C_{[0,1]}$, then

$$
\overline{\operatorname{dim}}_{B} \Gamma\left(D^{-v} f, I\right) \leq 2-v
$$

when $0<v<1$.

Proof. Let $0 \leq x<x+h \leq 1$. Then for $0<v<1$,

$$
\begin{aligned}
\left|D^{-v} f(x+h)-D^{-v} f(x)\right|= & \frac{1}{\Gamma(v)} \int_{0}^{x+h}(x+h-t)^{v-1} f(t) \mathrm{d} t-\frac{1}{\Gamma(v)} \int_{0}^{x}(x-t)^{v-1} f(t) \mathrm{d} t \mid \\
= & \frac{1}{\Gamma(v)} \mid \int_{0}^{x}\left[(x+h-t)^{v-1}-(x-t)^{v-1}\right] f(t) \mathrm{d} t \\
& +\int_{x}^{x+h}(x+h-t)^{v-1} f(t) \mathrm{d} t \mid \\
\leq & \frac{1}{\Gamma(v)}\left|\int_{0}^{x}\left[(x+h-t)^{v-1}-(x-t)^{v-1}\right] f(t) \mathrm{d} t\right| \\
& +\frac{1}{\Gamma(v)}\left|\int_{x}^{x+h}(x+h-t)^{v-1} f(t) \mathrm{d} t\right| \\
:= & I_{1}+I_{2} .
\end{aligned}
$$

Since $f(x) \in C_{[0,1]}$, there exists a positive number $M$ such that $|f(x)| \leq M$ for $\forall x \in I$. For $I_{1}$ defined above, it holds

$$
\begin{aligned}
I_{1} & \leq \frac{1}{\Gamma(v)} \int_{0}^{x}\left|(x+h-t)^{v-1}-(x-t)^{v-1}\right| \cdot|f(t)| \mathrm{d} t \\
& \leq \frac{M}{\Gamma(v)} \int_{0}^{x}\left[(x-t)^{v-1}-(x+h-t)^{v-1}\right] \mathrm{d} t \\
& =\frac{M}{v \Gamma(v)}\left[x^{v}+h^{v}-(x+h)^{v}\right] \\
& \leq \frac{2 M}{\Gamma(v+1)} h^{v} .
\end{aligned}
$$

Meanwhile,

$$
\begin{aligned}
I_{2} & \leq \frac{1}{\Gamma(v)} \int_{x}^{x+h}(x+h-t)^{v-1}|f(t)| \mathrm{d} t \\
& \leq \frac{M}{\Gamma(v)} \int_{x}^{x+h}(x+h-t)^{v-1} \mathrm{~d} t \\
& \leq \frac{M}{\Gamma(v+1)} h^{v} .
\end{aligned}
$$

Thus, we have

$$
I_{1}+I_{2} \leq C h^{v}
$$

This means

$$
\left|D^{-v} f(x+h)-D^{-v} f(x)\right| \leq C h^{v} .
$$

So, we know

$$
D^{-v} f(x) \in C_{[0,1]}^{v} .
$$


From Lemma 2.1,

$$
\overline{\operatorname{dim}}_{B} \Gamma\left(D^{-v} f, I\right) \leq 2-v
$$

Conclusion of Theorem 2.2 holds.

From Theorem 2.2, upper Box dimension of Riemann-Liouville fractional integral of order $v$ of any continuous functions $f(x)$ on $I$ is no more than $2-v$ when $0<v<1$. If Box dimension of $D^{-v} f(x)$ exists,

$$
\operatorname{dim}_{B} \Gamma\left(D^{-v} f, I\right) \leq 2-v, \quad 0<v<1 .
$$

With Definition 1.1, we know

$$
1 \leq \operatorname{dim}_{B} \Gamma\left(D^{-v} f, I\right) \leq 2-v, \quad 0<v<1
$$

If $v=1$, it is obvious that Box dimension of $D^{-v} f(x)$ exists and

$$
\operatorname{dim}_{B} \Gamma\left(D^{-v} f, I\right)=1
$$

for any $f(x) \in C_{[0,1]}$. In fact, $D^{-1} f(x)$ is a differentiable function on $I$, then it is of bounded variation. By Ref. [4], we know (2.1) holds.

In Theorem 2.2, there is a condition that the orders of Riemann-Liouville fractional integral belong to $(0,1)$. From the definition of Riemann-Liouville fractional calculus and Theorem 2.2, we can get the following result.

Corollary 2.3. Let $f(x) \in C_{[0,1]}$.

(1) If $0<v<1$,

$$
\overline{\operatorname{dim}}_{B} \Gamma\left(D^{-v} f, I\right) \leq 2-v .
$$

(2) If $v \geq 1$,

$$
\operatorname{dim}_{B} \Gamma\left(D^{-v} f, I\right)=1
$$

If $\operatorname{dim}_{B} \Gamma(f, I)=s$ and $2-v \leq s$, upper Box dimension of Riemann-Liouville fractional integral of $f(x)$ is no more than Box dimension of $f(x)$. In the next section, we will prove upper Box dimension of Riemann-Liouville fractional integral of a type of continuous functions is no more than Box dimension of continuous functions themselves.

\section{Upper Bound of Upper Box Dimension of Riemann-Liouville Fractional Integral of $f(x) \in C_{[0,1]}^{\alpha}$}

Let $f(x) \in C_{[0,1]}^{\alpha}$. We discuss upper bound of upper Box dimension of Riemann-Liouville fractional integral of $f(x)$ when $0<v<1$. If $2-v \leq \operatorname{dim}_{B} \Gamma(f, I)=2-\alpha$, we can still get upper bound of upper Box dimension of Riemann-Liouville fractional integral of $f(x)$ is no more than Box dimension of $f(x)$ by another method which is the following Theorem 3.1.

Theorem 3.1. Let $f(x) \in C_{[0,1]}^{\alpha}$ and $2-v \leq \operatorname{dim}_{B} \Gamma(f, I)=2-\alpha$. Then

$$
\overline{\operatorname{dim}}_{B} \Gamma\left(D^{-v} f, I\right) \leq 2-\alpha
$$

when $0<v<1$. 
Proof. Let $0 \leq x<x+h \leq 1$. We calculate difference between $D^{-v} f(x+h)$ and $D^{-v} f(x)$ when $0<v<1$. That is,

$$
\begin{aligned}
& D^{-v} f(x+h)-D^{-v} f(x)= \frac{1}{\Gamma(v)} \int_{0}^{x+h}(x+h-t)^{v-1} f(t) \mathrm{d} t \\
&-\frac{1}{\Gamma(v)} \int_{0}^{x}(x-t)^{v-1} f(t) \mathrm{d} t \\
&= \frac{1}{\Gamma(v)}\left[\int_{0}^{x+h}(x+h-t)^{v-1} f(t) \mathrm{d} t-\int_{0}^{x}(x-t)^{v-1} f(t) \mathrm{d} t\right] \\
&:=I_{3} .
\end{aligned}
$$

Let $t=y(x+h)$. So

$$
\begin{aligned}
I_{3} & =\frac{1}{\Gamma(v)}\left[\int_{0}^{x+h}(x+h-y(x+h))^{v-1} f(y(x+h)) \mathrm{d} y(x+h)-\int_{0}^{x}(x-t)^{v-1} f(t) \mathrm{d} t\right] \\
& =\frac{1}{\Gamma(v)}\left[\int_{0}^{1}(x+h)^{v}(1-y)^{v-1} f(y(x+h)) \mathrm{d} y-\int_{0}^{x}(x-t)^{v-1} f(t) \mathrm{d} t\right] .
\end{aligned}
$$

Let $t=y x$. Then,

$$
\begin{aligned}
I_{3} & =\frac{1}{\Gamma(v)}\left[\int_{0}^{1}(x+h)^{v}(1-y)^{v-1} f(y(x+h)) \mathrm{d} y-\int_{0}^{x}(x-y x)^{v-1} f(y x) \mathrm{d} y x\right) \\
& =\frac{1}{\Gamma(v)}\left(\int_{0}^{1}(x+h)^{v}(1-y)^{v-1} f(y(x+h)) \mathrm{d} y-\int_{0}^{1} x^{v}(1-y)^{v-1} f(y x) \mathrm{d} y\right) \\
& =\frac{1}{\Gamma(v)} \int_{0}^{1}(1-t)^{v-1}\left[(x+h)^{v} f(t(x+h))-x^{v} f(t x)\right] \mathrm{d} t .
\end{aligned}
$$

Write $F_{x}(t)=x^{v} f(t x)$. We have

$$
I_{3}=\frac{1}{\Gamma(v)} \int_{0}^{1}(1-t)^{v-1}\left[F_{x+h}(t)-F_{x}(t)\right] \mathrm{d} t .
$$

If $f(x)$ satisfies Hölder condition defined as Definition 1.3, it holds

$$
\begin{aligned}
\left|I_{3}\right| & =\frac{1}{\Gamma(v)}\left|\int_{0}^{1}(1-t)^{v-1}\left[F_{x+h}(t)-F_{x}(t)\right] \mathrm{d} t\right| \\
& =\frac{1}{\Gamma(v)}\left|\int_{0}^{1}(1-t)^{v-1}\left[F_{x+h}(t)-x^{v} f(t(x+h))+x^{v} f(t(x+h))-F_{x}(t)\right] \mathrm{d} t\right|
\end{aligned}
$$

Since $|f(x)| \leq M$, then

$$
\begin{aligned}
\left|I_{3}\right| \leq & \frac{1}{\Gamma(v)} \int_{0}^{1}(1-t)^{v-1}\left|(x+h)^{v} f(t(x+h))-x^{v} f(t(x+h))\right| \mathrm{d} t \\
& +\frac{1}{\Gamma(v)} \int_{0}^{1}(1-t)^{v-1} x^{v}|f(t(x+h))-f(t x)| \mathrm{d} t \\
\leq & \frac{M}{v \Gamma(v)} h^{v}+\frac{1}{v \Gamma(v)} C h^{\alpha} \\
= & \frac{1}{\Gamma(v+1)}\left(M h^{v}+C h^{\alpha}\right) .
\end{aligned}
$$

By Definition 1.1 and Lemma 2.1,

$$
\overline{\operatorname{dim}}_{B} \Gamma\left(D^{-v} f, I\right) \leq \max \{2-v, 2-\alpha\} .
$$


Thus, we know upper Box dimension of Riemann-Liouville fractional integral of $f(x)$ is no more than Box dimension of $f(x)$ itself.

Similar with Theorem 2.2, Theorem 3.1 gives an upper bound of upper Box dimension of RiemannLiouville fractional integral functions. We don't know that upper Box dimension of Riemann-Liouville fractional integral functions is no more than Box dimension of functions themselves or not. But we have a better conclusion as the following theorem.

Theorem 3.2. Let $f(x) \in C_{[0,1]}^{\alpha}$ and $f(0)=0$. Then

$$
\overline{\operatorname{dim}}_{B} \Gamma\left(D^{-v} f, I\right) \leq \min \{2-v, 2-\alpha\}
$$

when $0<v<1$.

Proof. Let $0 \leq x<x+h \leq 1$ and $0<v<1$. It holds

$$
\begin{aligned}
D^{-v} f(x+h)-D^{-v} f(x)= & \frac{1}{\Gamma(v)} \int_{0}^{x+h}(x+h-t)^{v-1} f(t) \mathrm{d} t \\
& -\frac{1}{\Gamma(v)} \int_{0}^{x}(x-t)^{v-1} f(t) \mathrm{d} t \\
= & \frac{1}{\Gamma(v)} \int_{0}^{x+h}(x+h-t)^{v-1} f(t) \mathrm{d} t \\
& -\frac{1}{\Gamma(v)} \int_{h}^{x+h}(x+h-t)^{v-1} f(t-h) \mathrm{d} t \\
= & \frac{1}{\Gamma(v)} \int_{h}^{x+h}(x+h-t)^{v-1}[f(t)-f(t-h)] \mathrm{d} t \\
& +\frac{1}{\Gamma(v)} \int_{0}^{h}(x+h-t)^{v-1} f(t) \mathrm{d} t \\
:= & I_{4}+I_{5} .
\end{aligned}
$$

Since $|f(x)-f(y)| \leq C|x-y|^{\alpha}$ holds for any $x, y \in I$,

$$
\left|I_{4}\right| \leq \frac{C}{v \Gamma(v)} h^{\alpha} .
$$

Meanwhile $f(0)=0$, so we have

$$
\left|I_{5}\right| \leq \frac{C}{v \Gamma(v)} h^{\alpha} \cdot h^{v}
$$

Thus,

$$
\left|D^{-v} f(x+h)-D^{-v} f(x)\right| \leq C h^{\alpha} .
$$

By Lemma 2.1,

$$
\overline{\operatorname{dim}}_{B} \Gamma\left(D^{-v} f, I\right) \leq 2-\alpha .
$$

This shows upper Box dimension of Riemann-Liouville fractional integral of any continuous funcitons satisfying Hölder condition is non-increasing. Furthermore, according to Theorem 2.2, we have

$$
\overline{\operatorname{dim}}_{B} \Gamma\left(D^{-v} f, I\right) \leq \min \{2-v, 2-\alpha\} .
$$

Theorem 3.1 shows there exists certain type of continuous functions whose upper Box dimension of Riemann-Liouville fractional integral is no more than $2-\alpha$ when $0<v<1$. Furthermore, we have the following conclusion.

Corollary 3.3. If $f(x) \in C_{[0,1]}^{\alpha}, f(0)=0$ and

$$
\operatorname{dim}_{B} \Gamma(f, I)=2-\alpha
$$


then, upper bound of upper Box dimension of Riemann-Liouville fractional integral is no more than Box dimension of $f(x)$ itself.

Upper bound of upper Box dimension of Riemann-Liouville fractional integral of certain functions defined as Corollary 3.3 seems not increase. If Box dimension of both a continuous function $f(x)$ and its Riemann-Liouville fractional integral function $D^{-v} f(x)$ exist when $0<v<1$, we can get

$$
\operatorname{dim}_{B} \Gamma\left(D^{-v} f, I\right) \leq \operatorname{dim}_{B} \Gamma(f, I), \quad 0<v<1
$$

Remark 3.4. From discussion above, we find a type of continuous functions whose Riemann-Liouville fractional integral functions fractal dimension is no more than fractal dimension of functions themselves. We think fractal dimension of Riemann-Liouville fractional integral of all continuous functions is no more than fractal dimension of themselves.

Furthermore, the relationship between fractal dimension of Riemann-Liouville fractional integral of certain continuous functions and fractal dimension of functions themselves is linear. A famous example maybe Weierstrass function. In other words, fractal dimension of Weierstrass function from fractal dimension of Riemann-Liouville fractional integral of Weierstrass function is equal to order of RiemannLiouville fractional integral.

With Definition 1.2 and discussion above, we have the following theorem.

Theorem 3.5. If $f(x) \in C_{[0,1]}^{\alpha}$ and

$$
\operatorname{dim}_{B} \Gamma(f, I)=s,
$$

then

$$
\overline{\operatorname{dim}}_{B} \Gamma\left(D^{-v} f, I\right) \leq s
$$

holds for any $v>0$.

If Box dimension of both $D^{-v} f(x)$ and $f(x)$ exist,

$$
\operatorname{dim}_{B} \Gamma\left(D^{-v} f, I\right) \leq \operatorname{dim}_{B} \Gamma(f, I)
$$

holds for any $v>0$.

With the following definition of count $\alpha$-Hölder condition, we give Corollary 3.7.

Definition 3.6.[1] Suppose that there are numbers $\delta_{0}>0,0<\alpha<1$ with the following property: for each $x \in I$ and $0<\delta \leq \delta_{0}$ there exists $y$ such that $|x-y| \leq \delta$ and

$$
|f(x)-f(y)| \geq C \delta^{\alpha}
$$

Then

$$
\underline{\operatorname{dim}}_{B} \Gamma(f, I) \geq 2-\alpha .
$$

If $f(x)$ satisfies condition of Definition 3.6, we say $f(x)$ satisfies count $\alpha$-Hölder condition and write it as $f(x) \in C_{[0,1]}^{-\alpha}$. It is easy to get the following conclusion.

Corollary 3.7. If $f(x) \in C_{[0,1]}^{\alpha} \cap C_{[0,1]}^{-\alpha}$,

$$
\overline{\operatorname{dim}}_{B} \Gamma\left(D^{-v} f, I\right) \leq \operatorname{dim}_{B} \Gamma(f, I) .
$$

Thus we find a class of fractal functions without expressions whose upper Box dimension of RiemannLiouville fractional integral is no more than Box dimension of functions themselves.

Acknowledgments. research is supported by National Natural Science Foundation of China(Grant No. 11201230, 11271182) and Natural Science Foundation of Jiangsu Province(Grant No. BK20161492). 


\section{References}

1. J. Falconer, Fractal geometry: mathematical foundations and applications, John Wiley Sons Inc., Newyork. 1990.

2. Y. S. Liang, The relationship between the Box dimension of the Besicovitch functions and the orders of their fractional calculus, Applied Mathematics and Computation 200 (2008), 197-207.

3. Y. S. Liang, On the fractional calculus of Besicovitch function, Chaos, Solitons and Fractals 42 (2009), 2741-2747.

4. Y. S. Liang, Box dimensions of Riemann-Liouville fractional integrals of continuous functions of bounded variation, Nonlinear Analysis 72 (2010), 4304-4306.

5. Y. S. Liang, Some remarks on continuous functions of unbounded variation. Acta Mathematica Sinica, Chinese Series 59 (2016), 215-232.

6. K. B. Oldham, J. Spanier, The fractional calculus, Academic Press, Newyork, 1974.

7. B. Ross, The fractional calculus and its applications, Springer-Verlag, Berlin, Heidelberg, 1975.

8. H. J. Ruan, W. Y. Su, K. Yao, Box dimension and fractional integral of linear fractal interpolation functions, Journal of Approximation Theory 161 (2009), 187-197.

9. F. B. Tatom, The relationship between fractional calculus and fractals, Fractals 3 (1995), 217-229.

10. Z. Y. Wen, Mathematical foundations of fractal geometry, Science Technology Education Publication House, Shanghai, 2000[in Chinese].

11. K. Yao, W. Y. Su, S. P. Zhou, On the fractional calculus of a type of Weierstrass function, Chinese Annals of Mathematics 25(A) (2004), 711-716.

12. K. Yao, W. Y. Su, S. P. Zhou, On the fractional derivatives of a fractal function, Acta Mathematica Sinica, English Series 22 (2006), 719-722.

13. M. Zähle, H. Ziezold, Fractional derivatives of Weierstrass-type functions, Journal of Computational and Applied Mathematics 76 (1996), 265-275.

14. S. P. Zhou, Fractional integrals of the Weierstrass functions: The exact Box dimension, Analysis in Theory and Applications. 20 (2004), 332-241. 